\title{
Effect of signal satiation on signal detection in auditory vigilance
}

\section{J. P. DAS, University of Alberta, Edmonton, Canada, and} PRATIBHA PRADHAN, Ravenshaw College, Cuttack. India

Sixty Ss in a vigilance task were required to detect the appearance of three odd numbers in succession in a random series of numbers between 1 and 9. Following a 30-min perfornance, half of them were satiated on the five odd numbers between 1 and 9 whereas the other half had an irrelevant task. Immediately afterwards, both groups took the vigilance task for 10 min. Results showed that the satiated group committed considerably more errors in signal detection than the nonsatiated group, and within the satiated group, the high satiators had poorer vigilance than the low satiators.

Decrement in vigilance performance has been attributed to perceptual fatigue by Broadbent (1958). Perceptual fatigue is a result of repeated stimulation, unlike motor fatigue which is a function of repeated response. In a typical auditory vigilance task used in the present experiment, $S$ is asked to listen to a random series of numbers from 1 to 9 and to detect the occurrence of three consecutive instances of odd numbers. Deterioration in signal detection is usually noticed within 30 min (Das, 1964), which may be regarded as the result of accumulation of perceptual fatigue.

In the present study, we have examined the effect of stimulus repetition through the technique of semantic satiation (Lambert \& Jakobovits, 1960) on vigilance performance. The technique involves the repetition of a stimulus (number or word) as fast as possible by $S$ for at least 15 sec. Das (1968) assumes that this leads to a decrement in attention paid to the stimulus immediately following repetition; such inconsequential repetition of a meaningful stimulus is hypothesized to bring about an extinction of orienting response (Sokolov, 1960) normally evoked by the stimulus.

\section{Subjects}

Sixty university students, males, aged between 20 and 25 years with a median age of 22 were employed as Ss. They were taking Master's level courses in areas other than Psychology in an Indian University.

Task

The vigilance task was a $1 / 2$-h continuous series of numbers 1 to 9 randomly recorded on magnetic tape and played back to the S. The digits occurred approximately at the rate of 30 per min. Signals (three odd digits occurring in a row) per each block of $5 \mathrm{~min}$ were between 6 and 8 , with a variable interval of 10 to $62 \mathrm{sec}$ between two successive signals. Ss were tested individually, and were instructed to tap a key every time they heard a sequence of three odd numbers. The $E$ maintained a record of the number of signals correctly detected and the number of false detections ( $S$ tapped the key when no signal had occurred) separately.

Design and Procedure

The Ss were randomly assigned to one of the two experimental treatments: satiation and nonsatiation conditions. In both conditions, the vigilance task was given for 30 min. This was followed by a 10-min period of satiation for one group, and an unrelated performance for $10 \mathrm{~min}$ by the other group.

On completion of the vigilance task, the Ss for the satiated condition were asked to rate the five odd digits on three 7 -point rating scales representing an evaluative dimension:
Good-Bad, Pleasant-Unpleasant. and Beautiful-Ugly (Osgood. Suci. \& Tannenbaum, 1957). When all the tive digits had been rated, the $S$ was presented one of the five odd digits, and was asked to repeat it aloud as fast as he conld for $1 \mathrm{~min}$. A repetition rate of 3 to 5 per sec was observed by the Ss. The digit, then, was rated again on the three scales. The same procedure was repeated until all five digits had been presented. This provided us with polarity-difference scores for satiation (Lambert \& Jakobovits, 1960). The satiation procedure at best took $10 \mathrm{~min}$.

The $\mathrm{Ss}$ in the nonsatiation condition were kept engaged in a neutral activity, putting pins in holes as fast as they could, for $10 \mathrm{~min}$. Our choice of this control task was guided by a desire to avoid even the remote possibility of decrement in attention to signal digits as might have happened had we taken a verbal task.

Both groups were again given the vigilance task for $10 \mathrm{~min}$, and as before, their omissions and false detections were recorded for the two 5-min periods.

\section{RESULTS AND DISCUSSION}

Figure 1 shows the percentage of omissions as well as the frequency of false detections for the two groups of Ss. The percentage of omissions (A) was calculated from the ratio of number of signals missed in a 5-min period to the number of signals presented in that period. (B) represents the total number of false detections for the group in a $5-\mathrm{min}$ period.

Vigilance

Examination of the first part of the graph shows a general increment in omissions, and hence decrement in vigilance during the $30-\mathrm{min}$ period for both the groups. The frequency of false detections, however, is high both at the initial and the final blocks, giving the trend of errors a bow-shape. The curves for false detections before the break are almost identical. False detections appear to be high initially, and at the end of the vigilance period when errors for omissions have increased. This does not seem to support the contention that false detections increase along with an increment in correct detection. Indeed, the opposite tendency is noticed in the nonsatiated group following a rest period. Incidentally, this finding provides evidence against a "guessing-correction" model. The model assumes that when the $S$ is unsure of having perceived the signal, he may be willing to guess, and since he will sometimes

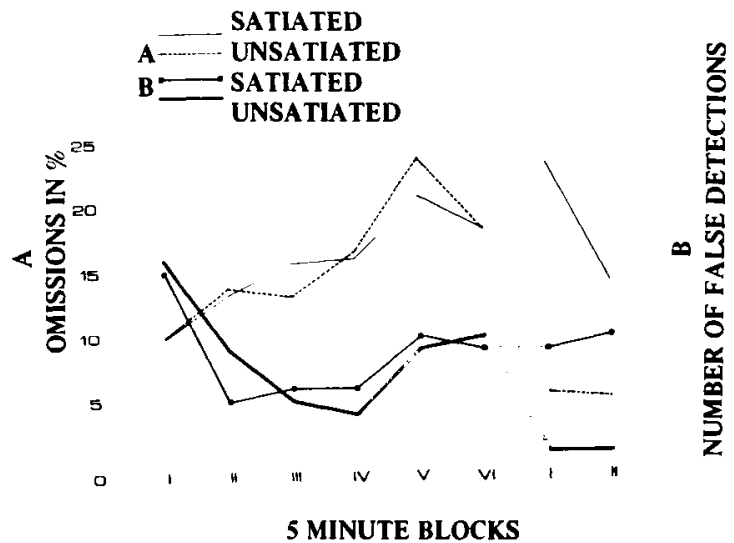

Fig. 1. Percentage of omissions (A) and frequency of false detections (B) of satiated and nonsatiated groups. 
guess correctly, this strategy will raise his rate of detection at the cost of a rise in the number of false detections (Buckner \& McGrath, 1963).

Effect of Satiation

Comparison between the performance of the two groups in the first $5 \mathrm{~min}$ following the break (second part of Fig. 1) shows a slight increment in omission errors for the satiated and a considerable decrement in these for the nonsatiated group. A $t$ test reveals this difference to be highly reliable $[t(29)=4.0$; $p<.011$. However, the satiated Ss show a decrement in omission errors in the next 5-min period. The number of false detections, too, decreases considerably for the nonsatiated group. One interpretation of these differences between the two groups may be in terms of motor fatigue-that for the satiated group, repetition was relatively more strenuous than the motor task was for the nonsatiated group. However, there are some objections against this. Since the nonsatiated group improved considerably in therr performance following the motor task, it would seem that the motor task was almost a "relaxing" experience, compared to the repetition of digits. On the other hand, it is unlikely that a considerable amount of motor fatigue was generated by a 1-min repetition of each of the five odd digits specially when the repetition periods were separated by nonstrenuous activities such as rating. Previous research on semantic satiation (Lambert \& Jakobovits, 1960) has shown that if there is any fatigue-like effect, it must be central rather than peripheral in nature, resembling closely Broadbent's notion of perceptual fatigue. In terms of perceptual fatigue, one would not require separate explanations for vigilance decrement and for the effect of semantic satiation on vigilance, since the accumulation of perceptual fatigue reduces vigilance prior to satiation and keeps it at that level following satiation due to repeated stimulus presentation. However, vigilance seems to improve after the first $5 \mathrm{~min}$ following satiation. An explanation in terms of extinction of OR may be more appropriate at this point. According to this, the satiated digits would get back their signal value when the vigilance task is resumed as a result of prior instruction; this would remove the effect of extinction, and reinstate ORs to the signal digits.

Presumably, Ss would vary in their strength of extinction of $O R$; hence the vigilance performance following satiation for the high satiators should be worse than that for the low satiators. We tested it by dividing the $30 \mathrm{Ss}$ of the satiated group into 14 high (mean satiation $=-7.0$ ) and 16 low (mean satiation $=+4.0$ ) satiators on the basis of their polaritydifference scores, and compared their mean errors of omissions. A $t$ test for the entire $10-$ min period of vigilance showed a higher number of omissions for the high satiators [means 3 vs $1.84 ; \mathrm{t}(28)=3.40 ; \mathrm{p}<.05$ ]. This finding provides some support for our hypothesis that semantic satiation may involve, primarily, a decrement of attention to satiated stimuli.

REFERENCES
BROADBENT, D. E. Perception and communication. New York: Pergamon Press, 1958.

BUCKNER, D. N., \& McGRATH, J. J. Vigilance: A symposium. New York: McGraw-Hill, 1963. Pp. 79-81.

DAS, J. P. Hypnosis, verbal satiation, vigilance, and some personality factors. Journal of Abnormal \& Social Psychology, 1964, 68, 72-78.

DAS, J. P. Semantic satiation and body-sway suggestibility. British Journal of Psychology, 1968, 59, 17-21.

LAMBERT, W. E., \& JAKOBOVITS, L. A. Verbal satiation and changes in the intensity of meaning. Journal of Experimental Psychology, $1960,60,376-383$.

OSGOOD, C. E., SuCI, G. J., \& TANNENBAUM, P. H. The measurement of meaning. Urbana: University of Illinois Press, 1957.

SOKOLOV, E. N. Neuronal models and the orienting reflex. In M. A. Brazier (Ed.), The central nervous system and behavior. New York: Josiah Macy Foundation, 1960. Pp. 187-276. 